\title{
Biliary peritonitis caused by a gallbladder injury due to low-energy blunt trauma
}

Received May 22, 2020

Revised May 29, 2020

Accepted May 31, 2020

Correspondence to

Jungchul Kim

Department of Trauma Surgery, Chonnam National University Hospital,

42 Jebong-ro, Dong-gu, Gwangju

61469 , Korea

Tel: +82-62-220-5442

Fax: +82-62-220-5445

E-mail: bluetholic@naver.com

\section{Hyunseok Jang, Younggoun Jo, Euisung Jeong, Min Gaegal, Yunchul Park, Jungchul Kim \\ Department of Surgery, Chonnam National University Hospital, Gwangju, Korea}

An isolated injury of the gallbladder after low-energy blunt abdominal trauma is rare. Here we report the case of a patient with biliary peritonitis caused by an impacted gallstone in the cystic plate after a simple slip-and-fall injury on the road.

Key Words: Gallstone, Cholecystectomy, Gallbladder, Laparotomy

\section{Introduction}

A gallbladder injury due to low-energy blunt abdominal trauma is rare. Here we report the case of a patient with biliary peritonitis caused by an impacted gallstone in the cystic plate after a gallbladder injury.

\section{Case presentation}

A 68-year-old woman was referred to the emergency department from an outside clinic after a simple slip-andfall injury. During the initial physical examination, the patient had mild abdominal pain. Her initial blood pressure was $130 / 80 \mathrm{mmHg}$, pulse rate was 80 beats/min, respiration rate was 20 breaths/min, and body temperature was $36.0^{\circ} \mathrm{C}$. Laboratory findings revealed a hemoglobin level of $12.1 \mathrm{~g} / \mathrm{dL}$, an aspartate aminotransferase/alanine transferase level of 166/237 U/L, and a total/direct bilirubin level of 1.74/0.33 mg/dL. Her white blood cell count was $12.4 \times 10^{3} / \mu \mathrm{L}$ (reference range: $4.8 \times 10^{3}-10.8 \times 10^{3}$ ), and her C-reactive protein level was $0.3 \mathrm{mg} / \mathrm{dL}$ (reference range:
0-0.3 mg/dL).

Abdominal computed tomography (CT) revealed mild traumatic injury to the liver (grade I according to the

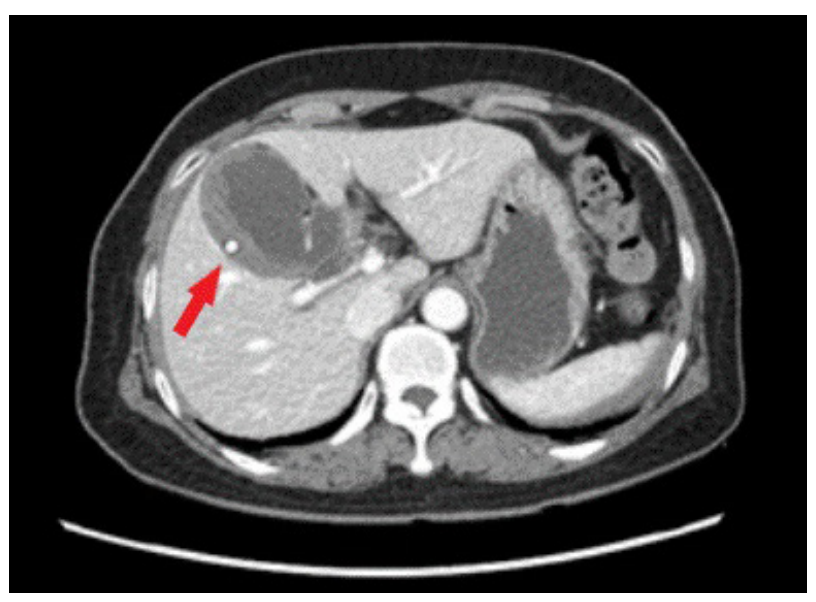

Fig. 1. Abdominal computed tomography performed in the emergency department. A small amount of hemoperitoneum is visible, and there is no evidence of active bleeding. Gallbladder stones are visible inside the gallbladder.

(c)This is an Open Access article distributed under the terms of the Creative Commons Attribution Non-Commercial License (http://creativecommons.org/licenses/by-nc/4.0) which permits unrestricted noncommercial use, distribution, and reproduction in any medium, provided the original work is properly cited.

Copyright (C) 2020 Korean Association for Research, Procedures and Education on Trauma. All rights reserved. 
American Association for the Surgery of Trauma classification system) and gallbladder, with a small amount of fluid in the perihepatic and perisplenic area (Fig. 1). Intra-abdominal fluids showed an attenuation of 40 Hounsfield units (HU), and the gallbladder wall showed no lesion.

On day 2 after admission and conservative treatment, the abdominal pain worsened and inflammatory markers levels were elevated. The patient's white blood cell count was $14.4 \times 10^{3} / \mu \mathrm{L}$, and her C-reactive protein level was 26.8 $\mathrm{mg} / \mathrm{dL}$. Follow-up abdominal CT revealed that the amount of collected fluid had increased, but no active bleeding was evident. Intra-abdominal fluids showed an attenuation of 10-15 HU, and a lesion was found in the gallbladder wall; therefore, bile peritonitis rather than hemoperitoneum was diagnosed (Fig. 2).

Owing to the patient's worsening abdominal pain, we decided to perform emergency surgery. In the operation theater, we observed collected fluid containing bile and bile leakage in the cystic plate area. A capsular tear with mild bleeding was detected at the cystic plate site. Moreover, during cholecystectomy, we discovered an impacted gallstone at the injury site. Biopsy findings revealed mucosal necrotizing cholecystitis with hemorrhage (Fig. 3). Furthermore, 9 days after the surgery, the patient was discharged without any complications.

\section{Discussion}

Blunt traumatic gallbladder injuries often manifest as contusions, perforations, or even avulsions (1). Risk factors of an isolated gallbladder injury should be considered. Laffey and Hay reported that gallbladder distension at the time of a traumatic event is a risk factor of an isolated
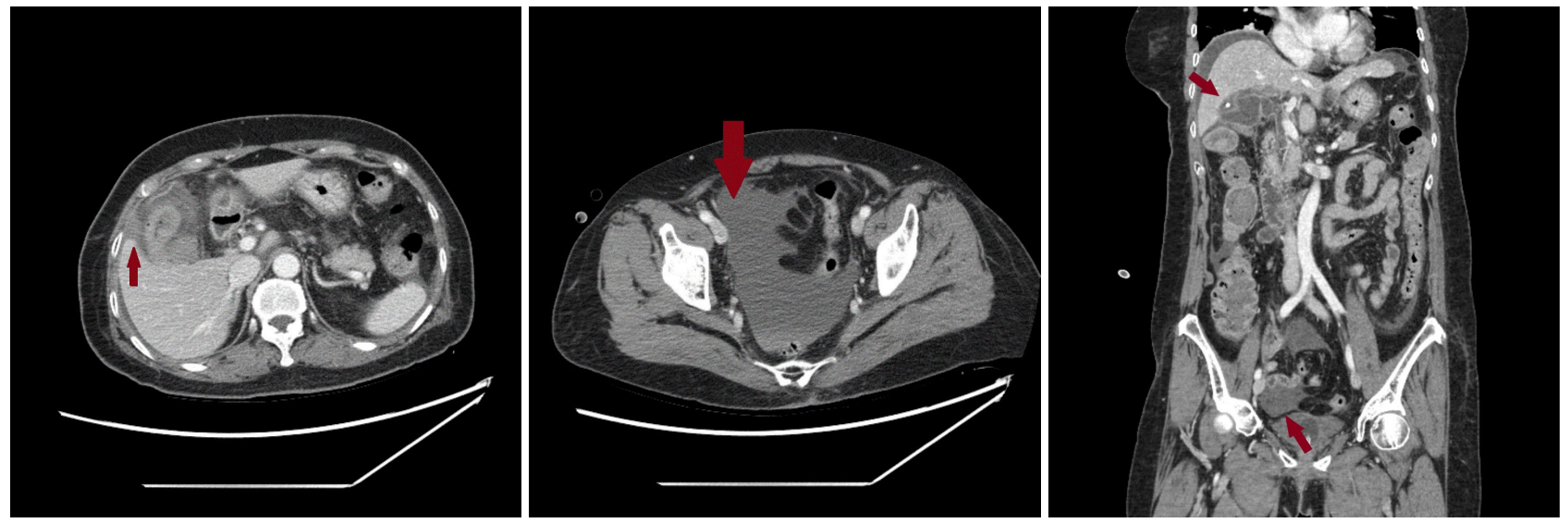

Fig. 2. Follow-up computed tomography on day 2 after admission. The amount of intra-abdominal fluid collection is moderately increased.
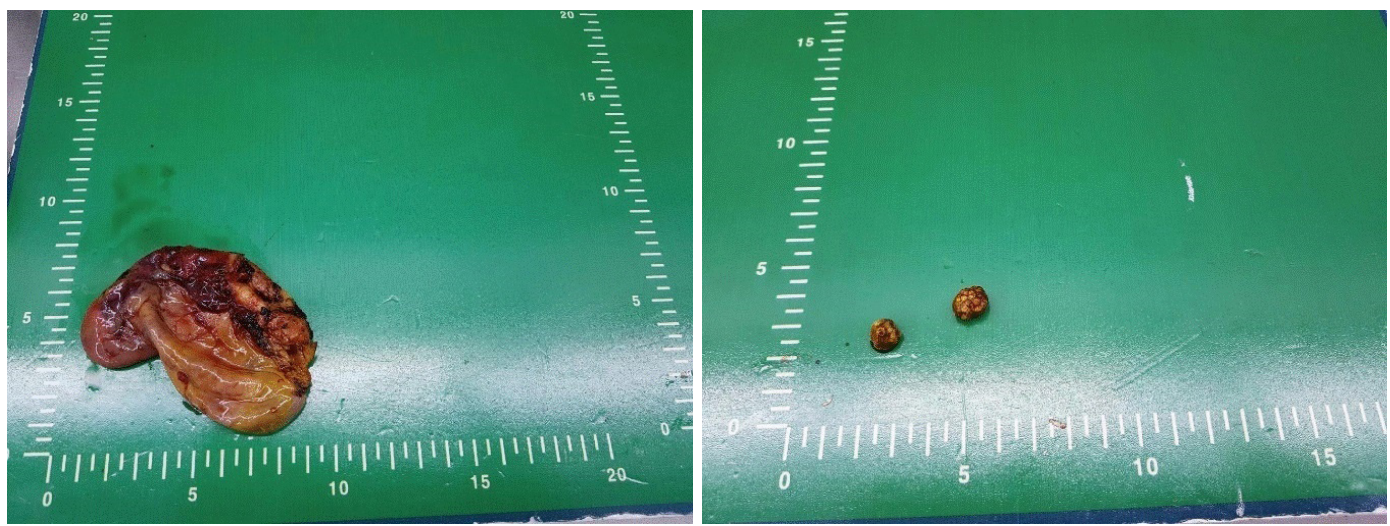

Fig. 3. Two calcified gallstones were extracted from within the gallbladder. 
injury. Distension can be caused by alcohol abuse, the after-meal state, or bile duct obstruction due to pre-existing gallbladder stones (2).

$\mathrm{CT}$ is sufficient for evaluating isolated gallbladder injuries, which are suspected when an ill-defined contour of the gallbladder wall, intraluminal hemorrhage, or luminal collapse is visible on CT. Blow-out lesions or abnormal fluid collection may indicate perforation or avulsion of the gallbladder. However, contusion injuries that result in minor injuries or delayed injuries are not easy to recognize (3).

In the present case, the patient might have had a pre-existing gallbladder injury at the time of arrival because the injury was difficult to recognize on initial CT-the amount of intra-abdominal fluid collection was small, and its attenuation on CT was $40 \mathrm{HU}$, leading to the possibility of hemoperitoneum.

These minor or delayed injuries can be identified if the patient's physical examination reveals signs of peritonitis, such as right upper quadrant abdomen tenderness, or if laboratory findings reveal abnormalities, such as increased levels of inflammatory markers. The final diagnosis is made using laparotomy, and treatment generally involves cholecystectomy (4).

Soderstrom et al. reported several cases of minor gallbladder contusion injuries that were nonsurgically managed (5). However, in such cases, the patients should be simultaneously monitored for complications of cholecystitis or delayed rupture.

When gallbladder injury is suspected, treatment and close follow-up are important because delayed diagnosis is related to poor prognosis. Furthermore, patients who exhibit more risk factors, such as gallbladder distension due to underlying conditions or the presence of gallstones, should be carefully monitored. If gallbladder injury is suspected, imaging follow-up and surgical intervention should be performed.

\section{Conflict of interest}

No potential conflict of interest relevant to this article was reported.

\section{References}

1. Zellweger R, Navsaria PH, Hess F, Omoshoro-Jones J, Kahn D, Nicol AJ. Gall bladder injuries as part of the spectrum of civilian abdominal trauma in South Africa. ANZ Journal of Surgery. 2005;75(7):559-61.

2. Laffey DA, Hay DJ. Isolated perforation of the gall bladder following blunt abdominal trauma. Postgrad Med J. 1979;55(641):212-4.

3. Erb RE, Mirvis SE, Shanmuganathan K. Gallbladder injury secondary to blunt trauma: CT findings. J Comput Assist Tomogr. 1994;18(5):778-84.

4. Sharma O. Blunt gallbladder injuries: presentation of twenty-two cases with review of the literature. J Trauma. 1995;39(3):576-80.

5. Soderstrom CA, Maekawa K, DuPriest RW Jr., Cowley RA. Gallbladder injuries resulting from blunt abdominal trauma: an experience and review. Ann Surg. 1981;193(1):60-6. 